\title{
Effect of next-nearest neighbor coupling on the optical spectra in bilayer graphene
}

\author{
A. R. Wright, ${ }^{1}$ Feng Liu, ${ }^{2}$ and C. Zhang ${ }^{1}$ \\ ${ }^{1}$ School of Engineering Physics, University of Wollongong, New South Wales 2552, Australia \\ ${ }^{2}$ Department of Materials Science and Engineering, \\ University of Utah, Salt Lake City, UT 84112, USA
}

\begin{abstract}
We investigate the dependence of the optical conductivity of bilayer graphene (BLG) on the intra- and inter-layer interactions using the most complete model to date. We show that the next nearest-neighbor intralayer coupling introduces new features in the low-energy spectrum that are highly sensitive to sample doping, changing significantly the "universal" conductance. Further, its interplay with interlayer couplings leads to an anisotropy in conductance in the ultraviolet range. We propose that experimental measurement of the optical conductivity of intrinsic and doped BLG will provide a good benchmark for the relative importance of intra- and inter-layer couplings at different doping levels.
\end{abstract}

PACS numbers: 73.50.Mx, 78.66.-w, 81.05.Uw

Since the isolation of single layers of graphite in 2003 [1], a lot of exciting work on single layer graphene (SLG) has been done [2]. For example, the prediction and observation of electron-hole symmetry and a half-integer quantum Hall effect [3, 4, 5], finite conductivity at zero chargecarrier concentration [3] , the strong suppression of weak localization [6, 7, 8], universal conductance [9, 10, 11] and magnetic enhancement of optical conductance in graphene nanoribbons [12].

More recently, attention has also been paid to SLG's cousin, bilayer graphene (BLG). The electronic and transport properties of BLG differ significantly from SLG in many respects, particularly at low energies in the 'Dirac' regime. Various models for low energy BLG exist in the literature depending on the coupling terms included, and whether electronic bands beyond the lowest energy subbands are retained [13, 14]. Many interesting results were obtained based on a model that includes only the most dominant of the interlayer coupling terms in BLG, as well as the usual nearest neighbor intralayer term [15]. By including the second most dominant interlayer coupling, some unusual properties such as a peculiar Landau-level spectrum have been derived [14], as well as a new low energy peak [16].

The 'universal conductance' of graphene is both a DC and an $\mathrm{AC}$ phenomenon. It is a direct result of the linear energy dispersion of graphene. Linear subbands imply both a constant density of states as well as consistent transition matrix elements, which means that for as long as the linear (Dirac) approximation is valid, the conductance is a constant. In the $\mathrm{AC}$ case, the value of the universal conductance of single layer graphene is $\sigma_{1}=e^{2} / 4 \hbar$. In the layered case, a standard benchmark is simply $\sigma_{n}=n \sigma_{1}$. However, this is not generally accurate, as the subband curvature caused by interlayer coupling in the case of layered graphene leads to a non-constant conductivity. This raises an important question: in what energy range is $\sigma_{n}=n \sigma_{1}$ applicable?

The infrared conductance of BLG has been measured by several groups [17, 18]. These results rely upon the effects of an induced gate voltage on the bandstructure, and all assume a discrepancy in onsite energy between the two layers. In ref. 17, Mak et al present the 'expected' IR conductance without the latter assumption, and find that it differs markedly from their experimental results. This demonstrates the need to assume an energetic discrepancy between the two layers in BLG. Our theoretical results, however, show a strong correlation to the results in ref.16 and 17, demonstrating that while an energetic discrepancy may exist, it is not necessary in describing the IR response observed experimentally.

In this letter, we study the dependence of the optical conductance of BLG on various intra- and inter-layer couplings. It is shown that the interplay of these couplings leads to a significant deviation in the behaviour of the conductance at low frequencies, which can, in turn, be tuned by electronic doping. In the important ultraviolet frequency band, this interplay leads to significant conductance anisotropy, i.e., the absorption along the armchair direction is around $50 \%$ stronger than that along the zigzag direction.

A typical BLG sheet consists of two SLG layers stacked in the orientation shown in Fig.1. Several forms of the Hamiltonian for BLG are used in the literature depending on the approximations used and the relative orientations of the two layers. The original consideration was given by Slonczewski-Weiss-McClure which included all three interlayer coupling terms 19, 20]. The most prominent interlayer term is the A-B and B-A coupling between sites which are directly above (or below) each other. Here we define this term as $\gamma_{1}=0.36 \mathrm{eV}$. The other two interlayer coupling terms are the A-B and B-A coupling between inequivalent sites which are not directly above or below each other, but offset by an amount $b=1.42 \dot{A}$, and the A-A and B-B terms which are similarly offset from one another, but represent equivalent sites in the SLG Brillouin Zone. These coupling terms are defined here as $\gamma_{3}=0.10 \mathrm{eV}$ and $\gamma_{4}=0.12 \mathrm{eV}$, respectively. We 


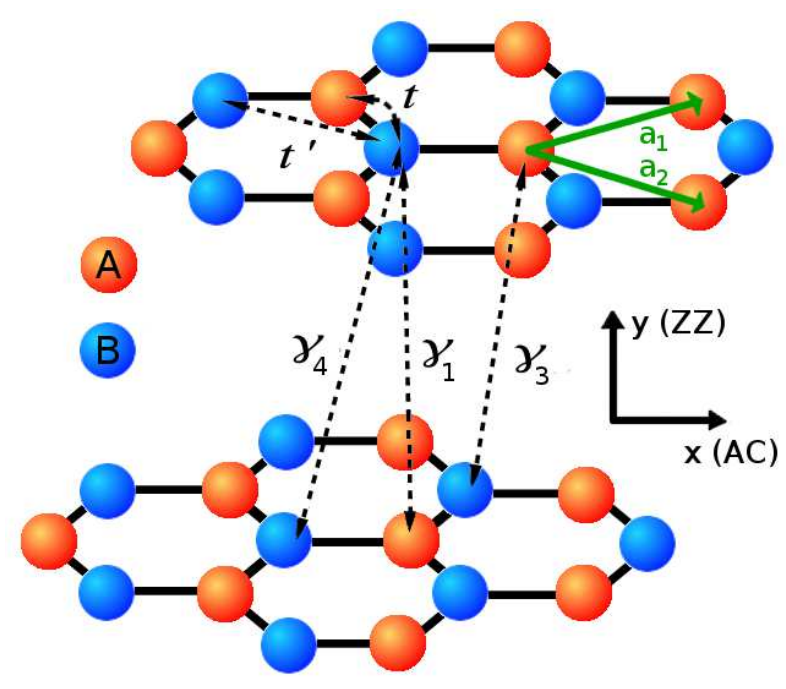

FIG. 1: The three interlayer and two intralayer coupling terms included in the BLG Hamiltonian. $\gamma_{3}$ and $\gamma_{4}$ differ in that they connect, respectively, inequivalent (eg. A-B) and equivalent (eg. A-A) points in the SLG Brillouin Zone. $\gamma_{1}$ is a directly vertical transition, and so the overlap of the wavefunctions is about $3 \times$ larger than $\gamma_{3}$ and $\gamma_{4}$. The armchair (AC) direction is given by the $\mathrm{x}$-axis, and the zig-zag (ZZ) direction is given by the $y$-axis. The lattice vectors $\mathbf{a}_{1}$ and $\mathbf{a}_{2}$ are also shown.

have also included the next nearest neighbor A-A and $\mathrm{B}-\mathrm{B}$ coupling which we define as $t^{\prime}=0.30 \mathrm{eV}$. Finally, as usual, the nearest neighbor A-B and B-A coupling is included, which is given here by $t=3.0 \mathrm{eV}$. All energies will be normalised relative to the first nearest neighbor coupling.

The full Hamiltonian matrix for the BLG system is

$$
H_{\mathrm{BLG}}=\left(\begin{array}{cccc}
t^{\prime} H^{\prime} & t H^{*} & \gamma_{4} H & \gamma_{1} \\
t H & t^{\prime} H^{\prime} & \gamma_{3} H^{*} & \gamma_{4} H \\
\gamma_{4} H^{*} & \gamma_{3} H & t^{\prime} H^{\prime} & t H^{*} \\
\gamma_{1} & \gamma_{4} H^{*} & t H & t^{\prime} H^{\prime}
\end{array}\right)
$$

where $H=e^{i k_{y} a / \sqrt{3}}\left(1+e^{i \mathbf{k} \cdot \mathbf{a}_{+}}+e^{i \mathbf{k} \cdot \mathbf{a}_{-}}\right)$and $H^{\prime}=$ $2\left(\cos \left(\mathbf{k} \cdot \mathbf{a}_{+}\right)+\cos \left(\mathbf{k} \cdot \mathbf{a}_{-}\right)+\cos \left(\mathbf{k} \cdot\left(\mathbf{a}_{+}-\mathbf{a}_{-}\right)\right)\right)$. Here $\mathbf{a}_{ \pm}=a\left( \pm \frac{1}{2},-\frac{\sqrt{3}}{2}\right)$ are the two lattice vectors shown in Fig.1. The eigenvalues and eigenvectors in the absence of $\gamma_{4}$ are readily solved. With $\gamma_{4}$ included however, the form of the solution is unwieldy. The eigenvalues in the simpler case are given by the (relatively) concise form

$$
\epsilon_{s, s^{\prime}}=t^{\prime}\left(\epsilon_{\mathrm{SLG}}^{2}-3\right)+s \sqrt{\epsilon_{S L}^{2}+\frac{\gamma_{12}^{+}}{2}+s^{\prime} \sqrt{\Gamma}}
$$

Where

$$
\Gamma=\epsilon_{S L}^{2} \gamma_{12}^{+}+\frac{\left(\gamma_{12}^{-}\right)^{2}}{4}+2 \gamma_{1} \gamma_{3} \epsilon_{S L}^{2} \operatorname{Re}(H)
$$

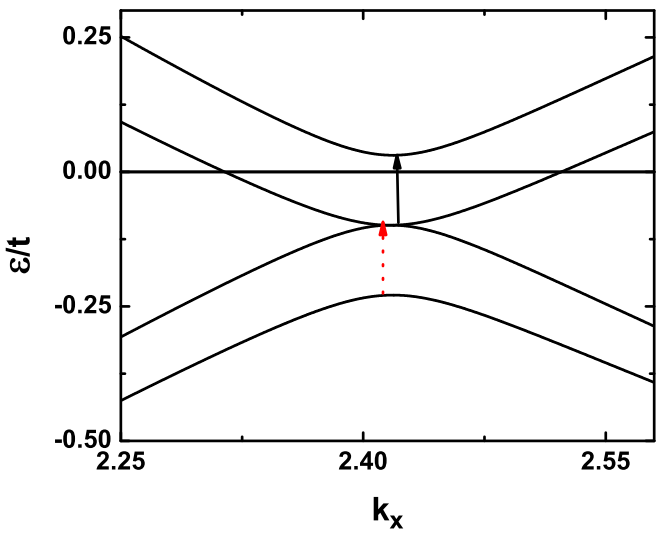

FIG. 2: The $k_{x}$ dependence of the bandstructure near the $\mathrm{K} / \mathrm{K}$ ' points. The red dashed arrow represents a transition which is permitted in an undoped sample if NNN coupling is neglected, but becomes forbidden when it is. The black solid arrow is the opposite: a previously forbidden transition becomes allowed when NNNs are included. The effect of doping is to raise or lower the Fermi level, making the inclusion of NNNs partly equivalent to doping.

And $\gamma_{12}^{ \pm}=\gamma_{3}^{2} \epsilon_{S L}^{2} \pm \gamma_{1}^{2}$, with $s, s^{\prime}= \pm 1$, and $\epsilon_{\mathrm{SL}}$ are the regular eigenvalues for the SLG system given as

$$
\begin{aligned}
\epsilon_{\mathrm{SLG}}= & t\left(1+4 \cos \left(a k_{x} / 2\right) \cos \left(a k_{y} / 2 \sqrt{3}\right)\right. \\
& \left.+4 \cos ^{2}\left(a k_{x} / 3\right)\right)^{\frac{1}{2}},
\end{aligned}
$$

From this result we see that there are two conduction bands and two valence bands which are confined above and below the line $\epsilon_{s, s^{\prime}}-t^{\prime}\left(\epsilon_{\mathrm{SLG}}^{2}-3\right)$, respectively. This simple result will form the basis for much of the discussion to follow.

The electron field operators can be constructed from the eigenvectors $\psi_{s, s^{\prime}}(\mathbf{k})$ such that $\Psi(\mathbf{r})=\left(1 / 4 \pi^{2}\right) \sum_{\mathbf{k}, s, s^{\prime}} a_{s, s^{\prime}}(\mathbf{k}) \psi_{s, s^{\prime}}(\mathbf{k}) e^{i \mathbf{k} \cdot \mathbf{r}}, \quad$ where $a_{s, s^{\prime}}(\mathbf{k}) \quad\left(a_{s, s^{\prime}}^{\dagger}(\mathbf{k})\right)$ denotes the annihilation (creation) operator for an electron in the $s$ or $s^{\prime}$ subband with momentum $\mathbf{k}$.

The band structure of BLG near the $\mathrm{K}$ points varies dramatically depending on the coupling terms included in the Hamiltonian. The effect of the various coupling terms are as follows:

The next nearest neighbor term $t^{\prime}$ defines the line about which the conduction and valence bands are confined, as can be seen in Fig.2. In the absence of lateral warping, the valence and conduction bands are symmetric about this line. Near the $\mathrm{K}$ points, the prefactor of the energy term associated with $t^{\prime}$ approaches 3 , and so the effect of this term is to shift all four bands downward by an amount $3 t^{\prime} \approx 0.1 \mathrm{eV}$. This can easily be seen from equation 2 , where $H^{\prime} \approx 3$. This breaks the electron-hole 


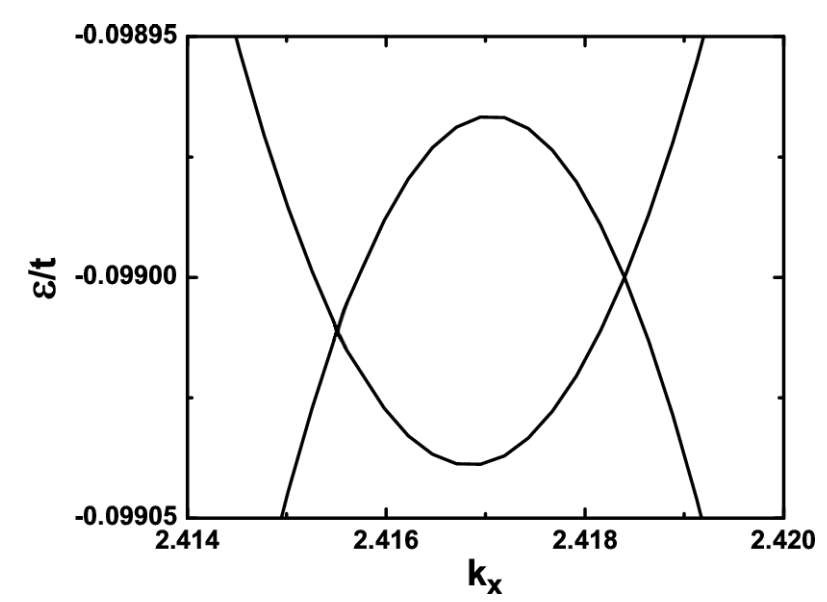

FIG. 3: The $k_{x}$ dependence of the two inner bands near the $\mathrm{K} / \mathrm{K}$ ' points zoomed right in to see the effects of the intralayer next nearest neighbors (NNN), and interlayer coupling terms $\gamma_{3}$ and $\gamma_{4}$. The NNN interaction has shifted these features well below the Fermi level. $\gamma_{3}$ causes a second dirac point to emerge, and $\gamma_{4}$ skews the bandstructure, causing one of the two Dirac points to be pushed down to a lower energy.

symmetry, but not the location of the minima in momentum space.

The term $\gamma_{1}$ represents the dominant interlayer $A-B$ and $B-A$ coupling. This term causes an energy gap to form between the two conduction bands, and an identical gap between the two valence bands of $\gamma_{1} \approx 0.13 \mathrm{eV} . \gamma_{1}$ also removes the linear dispersion at low energies. The electron hole symmetry is retained, and no lateral warping occurs. The effect of $\gamma_{1}$ is apparent in Fig.2.

The second interlayer coupling term $\gamma_{3}$ restores the linear lowest energy subband. This term causes what is usually referred to as 'trigonal warping' [14, 22]. A second set of Dirac points near the $\mathrm{K} / \mathrm{K}$ ' points emerges with $\gamma_{3}$ included, as can be seen in Fig.3.

Finally, $\gamma_{4}$ causes one of the Dirac points to be plunged below the NNN line, also seen in Fig.3. When the next nearest neighbor term couples with $\gamma_{4}$, however, the low energy $\mathrm{x}-\mathrm{y}$ isotropy is substantially weakened. While $\gamma_{3}$ causes the well known anisotropic 'trigonal warping', the energy range of this effect is in the order of $t / 10000 \approx 0.0003 \mathrm{eV}$. On its own, the effect of $\gamma_{4}$ is similarly small. Here, however, $\gamma_{4}$ and $t$ both couple equivalent sites, which causes a compounding of their individual effects on the electronic dispersion relation. The effect is quite a large deviation from isotropy. The effect of this deviation is most noticeable in the low energy conductance anisotropy shown in Fig.5.

We now evaluate the optical conductivity of BLG in the absence of disorder or impurities, over all relevant photon energies. By using the Kubo formula, the optical conductivity is given as [23],

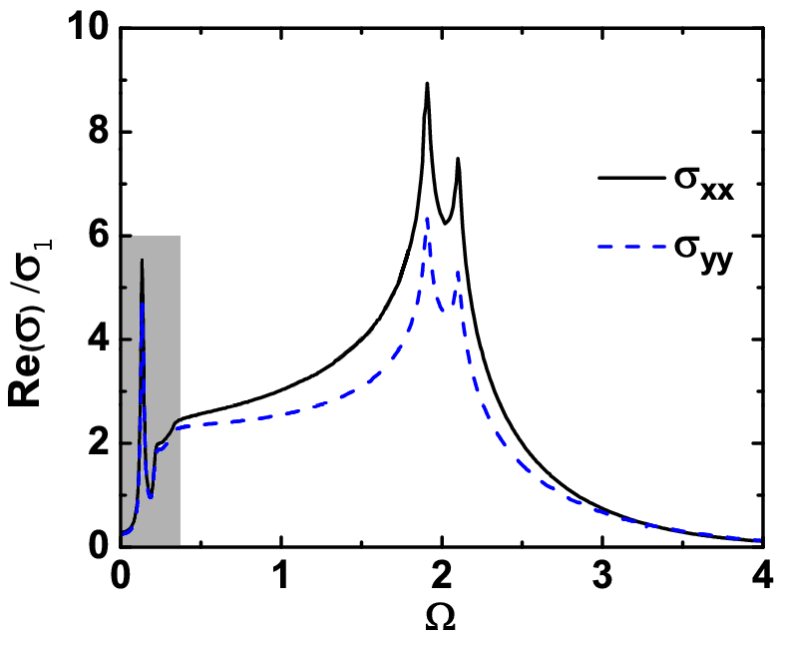

FIG. 4: The full energy optical conductance (in units of $\left.\sigma_{1}=e^{2} / \hbar\right)$ vs the normalized frequency $\Omega=\hbar \omega / t$ for bilayer graphene. Generally, $\sigma_{x x}$ (the armchair direction) has a larger optical response than $\sigma_{y y}$ (the zig-zag direction). When $\mathrm{NNN}$ and $\gamma_{4}$ are neglected, and at low energies, $\sigma_{x x}=\sigma_{y y}$. This is no longer the case here, with NNNs and $\gamma_{4}$ included. For $\epsilon<3 t^{\prime}$, there are no allowed transitions and the OC is approximately zero. The grey shaded area indicates the low energy region plotted in Fig.5.

$$
\sigma_{\mu, \nu}(\omega)=\frac{1}{\omega} \int_{0}^{\infty} \mathrm{d} t e^{i \omega t}\left\langle\left[J_{\mu}(t), J_{\nu}(0)\right]\right\rangle
$$

The components of the current operator can be calculated from $J_{\nu, \mu}(t)=e^{i H t} J_{\nu, \mu}(0) e^{-i H t}$, where $J_{\nu, \mu}(0)=$ $\Psi^{\dagger}(\mathbf{r}) \widehat{v}_{\nu, \mu} \Psi\left(\mathbf{r}^{\prime}\right)$, in which $\widehat{v}_{\nu, \mu}=\partial H / \partial k_{\nu, \mu}$, and $\nu, \mu=$ $x, y$. These values are calculated numerically, but we note that for each band there are three types of interband transitions, and also intraband transitions. In the case of no disorders and no intermediate interactions, it is found that intraband transitions cannot occur.

In Fig.4. we examine the full energy optical conductance of undoped bilayer graphene with NNN interactions included. Near the higher energy valley points, the optical conductivities exhibit two extrema, similar to the single peak found in single layer graphene [21, 24]. These peaks correspond to the two dominant vertical transitions between the two symmetric pairs of saddle points. The Joint Density of States in these valleys reaches a cusp-like maximum which leads to the extrema in the conductivities. These two energy peaks are separated by an amount $\hbar \omega=2 \gamma_{1}$, as expected from the bandstructure calculations.

Fig.5. shows the low energy optical conductivity of the undoped sample from the grey shaded region of Fig.4., as well as a sample doped to the level of the NNN interaction. The longitudinal conductivities vary greatly when including a non-zero NNN interaction. The effect of the dominant interlayer term $\gamma_{1}$ (ie. setting 


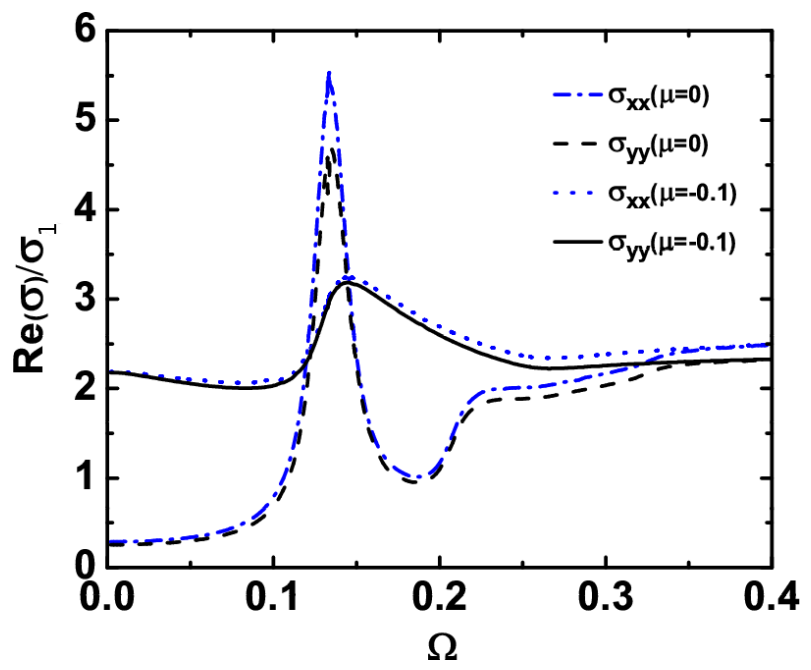

FIG. 5: The low energy optical conductance region from Fig.4. at two different doping levels. The black solid line is the OC of undoped BLG. The blue dotted line represents the $\mathrm{OC}$ at finite doping, and is equivalent to an undoped sample with no NNN interaction included which has been previously reported. The NNN- $\gamma_{4}$ coupling causes a new peak to emerge, and suppresses the previously reported one. This new peak is much larger and shifted to a lower photon energy. In a suitably doped sample, however, the $t^{\prime}=0$ (no NNN) peak has been retrieved by an effective shifting of the Fermi level.

$\gamma_{3}=\gamma_{4}=t^{\prime}=0$ ) at low energies has been reported recently [16]. This result has been retrieved in our result by doping the sample to $\mu=-0.1 \mathrm{eV} / \mathrm{t}$. In the undoped curve however, the previous result is entirely suppressed, and replaced by an approximately $2 \times$ larger, rounded peak, followed by a significant trough. This correlates well with the behaviour observed in IR experiments [17, 18], although without the added effects of an induced gate voltage. The doped case represents a transition of electrons from the upper conduction band to both the upper and lower valence bands, which is equivalent to neglecting the NNN coupling, as shown in Fig.2. The undoped case however reflects the suppression of transitions into the lower valence band, since it is already filled, and yet a new set of transitions occur between the two valence bands. These bands are separated by an approximately constant factor of $\gamma_{1}$, which leads to the large peak centred at $\hbar \omega \approx \gamma_{1}$ in the undoped bilayer case. The feature is in striking contrast with that of SLG. For SLG, the effect of the NNN coupling is to suppress the universal conductance at low frequencies [24]. As is clearly seen in Fig.5., for BLG the interplay of the interlayer coupling and the NNN coupling can suppress the conductance at low frequencies. However, it also induces a strong absorption peak in the far infrared before the onset of the universal conductance.

For this reason, the low energy approximations of the behaviour of bilayer graphene are generally more relevant to doped samples, with the undoped bilayer properties being drastically affected by the next nearest neighbor hopping and additional interlayer terms. Furthermore, when using existing theories to explain experimental results, it needs to be noted that an energetic discrepancy between layers, as well as the inclusion of a gate voltage, both cause some similar effects to the inclusion of the NNN interaction. All of these will therefore need to be accounted for when explaining any experimental result.

Furthermore, the conductance anisotropy observed in Figures 4 and 5, which is prominent even in the IR region when $\gamma_{4}$ and $t^{\prime}$ are both included, makes the polarization of the photon beam in experiments a relevant parameter. This orientation dependence of the optical conductance makes determination of the orientation of a BLG flake possible, and also makes BLG a potential partial polarizer. The doping dependence of the low energy conductance anisotropy makes this feature quite versatile.

Finally, as we have already mentioned, the value of the 'universal' conductivity is a topic of great interest at the moment. According to these results, which have been calculated from the most robust interlayer and intralayer model adopted to date, the value of the universal conductivity is $\sigma=2 \sigma_{1}$ where $\sigma_{1}=e^{2} / 4 \hbar$ is the universal optical conductivity of single layer graphene defined earlier. The range over which this value is applicable is greatly affected by the presence of the NNN interaction, and the electronic doping. In particular the NNN interaction causes the very low energy optical response to become negligible, and around the observed peak, the optical conductance is $\sigma_{\text {peak }} \approx 4 \sigma_{1}$.

In conclusion, we have studied the longitudinal optical conductivity of BLG with the inclusion of all relevant interlayer coupling terms and next nearest neighbor intralayer interactions. The optical conductivity exhibits double peak resonance separated by an amount $2 \gamma_{1}$ and centered around $\hbar \omega=2 t$. At low energies, the NNN interaction leads to entirely new behaviour of the optical conductivity. The results obtained without NNN coupling, however, can be retrieved by appropriate electronic doping. The interplay of the $\mathrm{NNN}-\gamma_{4}$ couplings were found to lead to significant low energy conductance anisotropy which is strongly doping dependent. Finally, the value of the universal conductivity with the most robust formalism used to date has also been determined, and is given by $\sigma_{2}=2 \sigma_{1}$. These results will be crucial to the experimental testing of accepted theories on bilayer graphene, and will be useful for potential low energy electronic and photonic applications of bilayer graphene.

This work is supported by the Australian Research Council. 
[1] K.S. Novoselov, A.K. Geim, S.V. Morozov, D. Jiang, Y. Zhang, S.V. Dubonos, I.V. Grigorieva, A.A. Firsov Science 306, 666 (2004).

[2] A. K. Geim and K. S. Novoselov, Nature Materials, 6, 183 (2007).

[3] K. S. Novoselov, A. K. Geim, S. V. Morozov, D. Jiang, M. I. Katsnelson, I. V. Grigorieva, S. V. Dubonos, and A. A. Firsov, Nature (London) 438, 197 (2005).

[4] Y. Zhang, Y.W. Tan, H.L. Stormer, and P. Kim, Nature (London) 438, 201 (2005).

[5] C. Berger, Z. Song, X. Li, X. Wu, N. Brown, C. Naud, D. Mayou, T. Li, J. Hass, A. N. Marchenkov, E. H. Konrad, P. N. First, and W. A. de Heer, Science 312, 1191 (2006).

[6] H. Suzuura and T. Ando, Phys. Rev. Lett. 89, 266603 (2002); J. Phys. Soc. Jpn. 72, 69 (2003).

[7] S.V. Morozov, K.S. Novoselov, M.I. Katsnelson, F. Schedin, L.A. Ponomarenko, D. Jiang, and A.K. Geim, Phys. Rev. Lett. 97, 016801 (2006).

[8] D.V. Khveshchenko, Phys. Rev. Lett. 97, 036802 (2006).

[9] V.P. Gusynin, S.G. Sharapov, and J.P. Carbotte, Phys. Rev. Lett. 96, 256802 (2006).

[10] A. B. Kuzmenko, E. van Heumen, F. Carbone, and D. van der Marel, Phys. Rev. Lett, 100, 117401 (2008).

[11] R. R. Nair, P. Blake, A. N. Grigorenko, K. S. Novoselov, T. J. Booth, T. Stauber, N. M. R. Peres, and A. K. Geim,
Science 320, 1308 (2008)

[12] J. Liu, A.R. Wright, C. Zhang, and Z. Ma, App. Phys. Lett., 93, 041106 (2008).

[13] J. Nilsson, A. H. Castro Neto, F. Guinea, and N. M. R. Peres, Phys. Rev. B, 78, 045405 (2008).

[14] E. McCann and V.I. Falko, Phys. Rev. Lett. 96, 086805 (2006)

[15] J. Nilsson, A. H. Castro Neto, N. M. R. Peres, and F. Guinea, Phys. Rev. B, 73, 214418 (2006).

[16] D. S. L. Abergel and V. I. Fal'ko, Phys. Rev. B 75, 155430(2007).

[17] Z. Q. Li,1, E. A. Henriksen, Z. Jiang, Z. Hao, M. C. Martin, P. Kim, H. L. Stormer, and D. N. Basov, Phys. Rev. Lett. 102, 037403 (2009).

[18] K. F. Mak, C. H. Lui, J. Shan, and T. F. Heinz1, arxiv 0905.0923

[19] J. W. McClure, Phys. Rev. 108, 612 (1957).

[20] J. C. Slonczewski and P. R. Weiss, Phys. Rev. 109, 272 (1958).

[21] C. Zhang, L. Chen and Z. Ma, Phys. Rev. B, 77, 241402 (2008).

[22] J. Cserti, A. Csordas, and G. David, Phys. Rev. Lett., 99, 066802 (2007).

[23] G. D. Mahan, "Many-Particle Physics" (Kluwer Academic, 2000).

[24] T. Stauber, N.M.R. Peres, and A.K. Geim, Phys. Rev. B., 78, 085432 (2008). 